\title{
Bone Health in Patients with Breast Cancer
}

\author{
An Expert Interview with Panagiota Andreopoulou \\ Division of Endocrinology, Department of Medicine, Weill Cornell Medicine, New York, NY, USA
}

DOl: https://doi.org/10.17925/OHR.2019.15.1.16

\section{Panagiota Andreopoulou}

Dr Panagiota Andreopoulou is an Attending Physician in the Division of Endocrinology, Department of Medicine at Weill Cornell Medicine, New York Presbyterian and an Assistant Professor of Medicine at the Weill Cornell Medical College. Prior to these appointments she held faculty positions at the Hospital for Special Surgery and at the Albert Einstein College of Medicine, Montefiore Medical Center. Her clinical and academic interests include osteoporosis, calcium and phosphate metabolism, and metabolic bone disorders. Dr Andreopoulou completed her Endocrinology fellowship at the National Institutes of Health in Bethesda, MD, where she trained at the National Institute of Diabetes, Digestive and Kidney Diseases and the National Institute of Dental and Craniofacial Research (NIDCR). She conducted clinical research at the Skeletal Clinical Studies Unit of the Craniofacial and Skeletal Diseases Branch of NIDCR. Her research focused on fibroblast growth factor 23 regulation, tumor-induced osteomalacia, hypoparathyroidism, and polyostotic fibrous dysplasia. She worked on clinical research protocols evaluating the utility of selective venous sampling and functional imaging in the management of tumor-induced osteomalacia and the effects of the treatment of hypoparathyroidism with synthetic human parathyroid hormone on bone parameters. These resulted in publications in peer-reviewed journals and an Endocrine Society Award for Oral Presentation. At Montefiore and at the Hospital for Special Surgery, Dr Andreopoulou was an associate investigator in multiple clinical research trials on therapies for neuroendocrine tumors and elucidation of factors affecting bone quality, respectively. Dr Andreopoulou is a member of the American Society for Bone and Mineral Research, the Endocrine Society, the American Association of Clinical Endocrinologists (AACE), the American College of Physicians, and the American Medical Women's Association. She is a member of the patient education committee of the AACE. Dr Andreopoulou produces and hosts a weekly radio broadcast at the Hellenic Public Radio Cosmos FM on health and wellness issues.

\section{Keywords}

Breast cancer, cancer treatment-induced bone loss, osteopenia, osteoporosis, skeletal health, bone density, fracture, aromatase inhibitors, Tamoxifen, bisphosphonates, denosumab

Disclosures: Panagiota Andreopoulou has nothing to disclose in relation to this article.

Review Process: This is an expert interview and as such, has not undergone the journal's standard peer review process.

Authorship: The named author meets the International Committee of Medical Journal Editors (ICMJE) criteria for authorship of this manuscript, takes responsibility for the integrity of the work as a whole, and has given final approval for the version to be published.

Received: February 13, 2019

Published Online: May 29, 2019

Citation: Oncology \& Hematology Review. 2019;15(1):16-9

Corresponding Author: Panagiota Andreopoulou, 211 East 80th Street, New York, NY 10075,

USA. E:paa2655@med.cornell.edu

\section{Support: No funding was received in}

the publication of this article.
$\mathrm{B}$ reast cancer and its treatment may have a negative effect on bone health. Some cancer therapies may lead to accelerated bone loss. Since breast cancer is a common condition that is often cured, the effects on bone health are a major concern for survivors as it can put them at risk of fractures that can significantly impact their quality of life.

\section{Q. What is the impact of breast cancer and its treatment on bone health?}

There is a very strong link between breast cancer and treatment for breast cancer, and skeletal deterioration and fracture risk. First of all, women with breast cancer are usually post-menopausal and thus have low bone mineral density (BMD) at baseline even prior to starting treatment. Furthermore, the great majority of women who start treatment for breast cancer often undergo an abrupt transition to a hormonal milieu that is associated with accelerated bone loss and increased risk of fractures for the duration of treatment as well as in their lifetime. The latter is true for both pre- and postmenopausal women. In the Women's Health Initiative study, breast cancer survivors had a 15\% increased risk of fractures compared with cancer-free women. ${ }^{1}$ Therapies used in the treatment of breast cancer that can accelerate bone loss include chemotherapy, hormone ablation therapy resulting in hypogonadism, antiestrogen therapies, radiation, and concurrent medications such as glucocorticoids. They render patients at risk for osteoporosis and fragility fractures that can compromise their quality of life and longevity.

Chemotherapy for breast cancer has been associated with lower BMD in postmenopausal ${ }^{2}$ and premenopausal women..$^{-5}$ In premenopausal women chemotherapy is often associated with ovarian dysfunction due to direct toxicity and interference with follicle maturation, meaning menopause can occur about 10 years earlier than it would naturally. ${ }^{3} 40 \%$ to $95 \%$ of women who receive chemotherapy experience premature ovarian insufficiency, depending on the regimen and their age.,5

Premature menopause due to ovarian suppression with gonadotropin-releasing hormone (GnRH) agonists, oophorectomy, or cytotoxic chemotherapy decreases circulating estrogen which accelerates bone resorption and decreases BMD. GnRH agonists act on gonadotropin receptors of the pituitary gland, reducing estrogen production. BMD decline is in the range of $3-7 \%$ in women on 
chemotherapy, ${ }^{6}$ 5\% on GnRH agonists, ${ }^{7}$ and as high as $18-19 \%$ over 2 years after oophorectomy. ${ }^{8}$ Short-term fractures are overall low in this younger, premenopausal population. However, these young women are treated with curative intent and expected to have decades of life expectancy after completing breast cancer therapy, hence this early significant bone loss is disadvantageous later in life.

Antiestrogen therapy with tamoxifen and aromatase inhibitors has been the mainstay of treatment in hormone receptor-positive breast cancer. Tamoxifen, a selective estrogen receptor modulator, has both agonist and antagonist effects on the estrogen receptor. It is an estrogen antagonist in the breast regardless of pre- or postmenopausal status, but also an estrogen agonist within the bone. In postmenopausal women tamoxifen protects against bone loss,, 90 with a $32 \%$ relative risk reduction in fractures compared with placebo. ${ }^{10}$ In contrast, tamoxifen decreases BMD in premenopausal women, even if they maintain regular menstrual cycles. ${ }^{9}$

Aromatase inhibitors such as letrozole, anastrozole, and exemestane block the conversion of androgen to estrogen, primarily in adipose tissue, by inhibiting the aromatase enzyme, thus profoundly decreasing serum estrogen levels in postmenopausal women. In premenopausal women an aromatase inhibitor may only be used with surgical or chemical ovarian ablation, as aromatase inhibitors do not affect ovarian estrogen production. In postmenopausal women adjuvant aromatase inhibitors are superior to tamoxifen and widely used. In those women, aromatase inhibitors accelerate bone loss and increase fracture risk by at least around 10\% $\%^{11-14}$ and up to double that (by 18-20\%) after 5 years of treatment. ${ }^{15}$ Longer duration of aromatase inhibitor therapy is associated with higher increases in the risk of fracture, at 14\% versus $9 \%$ in those treated for 10 years versus 5 years respectively. ${ }^{16}$

Local radiation therapy interrupts the vascular supply in the bone, and directly contributes to hypoxia, which inhibits osteoblast function and increases osteoclastogenesis. ${ }^{17}$ Radiation-related bone-specific toxicities include bone loss within the field of radiation, osteopenia or osteoporosis, increased risk of fracture, and avascular necrosis. ${ }^{18}$

\section{Q. What can be done to maintain bone health in patients with breast cancer who are receiving endocrine therapy?}

All women receiving treatment for breast cancer should be evaluated for their risk of fracture at the onset of therapy, particularly because osteoporosis may be a preexisting condition. Several bone and oncologic societies have published specific guidelines. 15,19 A thorough history and ideally a dual-energy X-ray absorptiometry (DXA) scan should be performed. Emphasis should be given to a healthy diet and normal weight. Adequate intake of dietary protein and natural antioxidants found in fruit and vegetables, rather than in supplements, is encouraged. We recommend avoiding excessive carbohydrates given recent indications that women with breast cancer on hormonal therapies may be at higher risk for developing diabetes, which in itself is a significant risk factor for fractures. In addition, habits detrimental to the bones such as smoking, excess caffeine, colored carbonated beverages, and alcohol, should be avoided.

The foundation of maintaining bone health includes intake of adequate calcium (1,000-1,200 mg daily, preferably from food sources; however, supplements may be added) and vitamin D 800-1,000 units/d for a 25-OH vitamin D level above $30 \mathrm{ng} / \mathrm{mL}$, as is recommended for fall and fracture prevention in the general osteoporosis population. ${ }^{20}$ Adequate calcium and vitamin $\mathrm{D}$ intake is important in decreasing the risk of hypocalcemia and maintaining bone mineralization in the setting of antiresorptive therapies.

Adequate weight-bearing and resistance exercise is also important and has been shown to limit bone loss in postmenopausal women with breast cancer, ${ }^{21}$ although fracture risk reduction has not been demonstrated. ${ }^{15}$

Lastly, medications that reduce bone resorption such as bisphosphonates and denosumab are widely used and indicated in these women as the only effective means to actually reduce fracture risk. In postmenopausal women with breast cancer receiving aromatase-inhibitor therapy, oral bisphosphonates (alendronate, ibandronate, and risedronate) when used up to 5 years are associated with increases in BMD and reduction in fracture risk..$^{15}$

The AZURE (Adjuvant Zoledronic Acid to Reduce Recurrence) trial investigated the addition of zoledronic acid to standard adjuvant treatments in patients with early breast cancer, over a treatment period of 5 years. The results showed that the addition of zoledronic acid significantly lowered overall fracture rate by $6.2 \%$ versus $8.3 \%$ in the control group. ${ }^{22}$ In the Z-FAST (Zometa-Femara Adjuvant Synergy Trial) and ZO-FAST (ZometaFemara Adjuvant Synergy Trial) studies, postmenopausal women on adjuvant aromatase-inhibitor therapy were randomized to either upfront use of zoledronic acid (4 mg every 6 months) or delayed use of the ZOL regimen. ${ }^{14,23}$ Both studies found that those treated with zoledronic acid upfront had increases in BMD, but fracture rates were not statistically different across the treatment arms.

Denosumab, $60 \mathrm{mg}$ every 6 months, is approved for women on aromatase inhibitors at high risk for osteoporotic fracture. The ABCSG-18 (Austrian Breast Cancer Study Group) trial of denosumab versus placebo ${ }^{24}$ demonstrated that time to first clinical fracture was decreased in the denosumab-treated group regardless of baseline BMD or age..$^{24} \mathrm{As}$ expected, denosumab was associated with increasing BMD at all skeletal sites.

\section{Q. What factors should be considered when including a bone-targeted agent in breast cancer therapy of postmenopausal women?}

The most important factors are the baseline BMD measurement along with the presence of certain co-morbidities that may affect adherence to treatment or increase the risk of adverse events. In a joint position statement from several societies, medical treatment is recommended in patients with a T-score less than -2.0 or if two or more clinical risk factors are present, including age $>65$ years old, low body mass index $(<20)$, smoking (current or history), personal history of fracture after 50 years of age, family history of hip fracture, glucocorticoid use of $>6$ months, and T-score less than -1.5. ${ }^{15,19}$ When T-scores are greater than -2.0 with no other risk factors, then monitoring for bone loss every 1-2 years is a reasonable option. ${ }^{15}$ Some experts support treatment of premenopausal women with antiresorptive therapy if they have undergone ovarian suppression and are receiving an aromatase inhibitor, with a T-score less than -1.0 , or with a prevalent vertebral fracture, although data are limited. ${ }^{25}$

Certain co-morbidities exclude certain therapies, for example kidney disease (bisphosphonates) and severe gastroesophageal reflux disease or dysphagia 
(oral bisphosphonates). Adherence to oral medications is a challenging issue in the treatment of osteoporosis in general. The anabolic agents teriparatide and abaloparatide, and agents containing estrogen, should be avoided in patients with breast cancer due to the concern for stimulating the growth of micrometastatic occult tumor cells and increasing the risk of breast cancer recurrence, as well as the potential increased risk of osteosarcoma in areas exposed to skeletal radiation. We do not have any data available regarding the efficacy and safety of a recently approved anti-sclerostin antibody with combined anabolic and antiresorptive effect (romosozumab) in women with breast cancer.

\section{Q. What data support the effectiveness of bisphosphonates and denosumab in reducing the risk of bone recurrences and breast cancer deaths in postmenopausal women?}

A meta-analysis by the Early Breast Cancer Trialists' Collaborative Group used individual patient data from all trials in early breast cancer that randomized patients to either bisphosphonates or control, and analyzed for cancer outcomes. ${ }^{26}$ In postmenopausal women $(n=11,767)$, treatment with a bisphosphonate was associated with a significant decrease in risk of breast cancer recurrence, distant recurrence, bone recurrence, and breast cancer mortality. Thus, experts generally recommend use of adjuvant bisphosphonates in postmenopausal women who are at high risk for breast cancer recurrence, ${ }^{27}$ although we are not yet certain of the exact mechanism of this phenomenon.

Adjuvant denosumab is being studied for potential anticancer effects. Preliminary results of the ABCSG-18 study reported in 2015 at the San Antonio Breast Cancer Symposium noted that the addition of denosumab to adjuvant aromatase inhibitors reduced the risk of breast cancer recurrence by 18\% ${ }^{28}$ However the DCARE (Study of Denosumab as Adjuvant Treatment of Women With High Risk Early Breast Cancer Receiving Neoadjuvant or
Adjuvant Therapy) study, which randomized women with breast cancer to adjuvant denosumab (120 mg every 6 months) or to placebo did not show an effect in disease-free survival and overall survival. ${ }^{29}$

\section{Q. What are the other advantages and risks of including bone-targeted therapies in breast cancer therapy?}

Bone targeted therapies are very effective in reducing fracture risk while also being very well tolerated with minimal, and generally reversible, adverse events. ${ }^{30-41}$ Therefore, their benefit to risk ratio is highly favorable despite the negative publicity that they have received in the lay press, regarding extremely rare adverse events such as osteonecrosis of the jaw or atypical femoral fractures..$^{42}$ Antiresorptives have been associated with osteonecrosis of the jaw, thus is exposed jaw bone with delayed healing in 1/300,000 patients and usually associated with another risk factor such as poor dental and oral hygiene, use of dentures, Vitamin D deficiency, use of steroids and invasive dental procedure such as extractions and implant post insertion..$^{43}$ Thus, good oral hygiene and routine dental care is encouraged for patients receiving this therapy. ${ }^{43}$ Another extremely rare adverse event associated with long-term treatment with antiresorptives is atypical subtrochanteric femoral fractures, which may limit the duration of treatment of bisphosphonates due to their accumulation in the skeleton, necessitating a drug holiday. Patients should be monitored for prodromal symptoms that usually

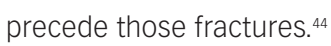

A recent concern is the rise in fracture risk upon discontinuation of denosumab. ${ }^{45}$ There are ongoing clinical trials assessing the protective effect of using a bisphosphonate subsequent to denosumab and this is often common practice nowadays in our patients.46-50 Overall, however, the benefits of antiresorptive agents in women with breast cancer, as well as the general population of women with osteoporosis, greatly outweigh the risks. $\square$
1. Chen $Z$, Maricic M, Aragaki AK, et al. Fracture risk increases after diagnosis of breast or other cancers in postmenopausal women: results from the Women's Health Initiative. Osteoporos Int. 2009:20:527-36.

2. Rodriguez-Rodriguez LM, Rodriguez-Rodriguez EM, OramasRodriguez JM, et al. Changes on bone mineral density after adjuvant treatment in women with non-metastatic breast cancer. Breast Cancer Res Treat. 2005;93:75-83.

3. Walshe JM, Denduluri N, Swain SM. Amenorrhea in premenopausal women after adjuvant chemotherapy for breast cancer. J Clin Oncol. 2006;24:5769-79.

4. Rizzoli R, Body JJ, Brandi ML, et al. Cancer-associated bone disease. Osteoporos Int. 2013;24:2929-53.

5. Kadakia KC, Henry NL. Adjuvant endocrine therapy in premenopausal women with breast cancer. Clin Adv Hematol Oncol. 2015;13:663-72.

6. Hadji P, Kauka A, Ziller M, et al. Effects of zoledronic acid on bone mineral density in premenopausal women receiving neoadjuvant or adjuvant therapies for HR+ breast cancer: the ProBONE II study. Osteoporos Int. 2014;25:1369-78.

7. Sverrisdottir A, Fornander $\mathrm{T}$, Jacobsson $\mathrm{H}$, et al. Bone mineral density among premenopausal women with early breast cancer in a randomized trial of adjuvant endocrine therapy. $J$ Clin Oncol. 2004;22:3694-99

8. Genant HK, Cann CE, Ettinger B, Gordan GS. Quantitative computed tomography of vertebral spongiosa: a sensitive method for detecting early bone loss after oophorectomy. Ann Intern Med. 1982;97:699-705.

9. Powles TJ, Hickish T, Kanis JA, et al. Effect of tamoxifen on bone mineral density measured by dual-energy $\mathrm{x}$-ray absorptiometry in healthy premenopausal and postmenopausal women. J Clin Oncol. 1996;14:78-84.

10. Fisher B, Costantino JP, Wickerham DL, et al. Tamoxifen for the prevention of breast cancer: current status of the National Surgical Adjuvant Breast and Bowel Project P-1 study J Natl Cancer Inst. 2005;97:1652-62.

11. Shapiro CL, Halabi S, Hars V, et al. Zoledronic acid preserves bone mineral density in premenopausal women who develop ovarian failure due to adjuvant chemotherapy: final results from CALGB trial 79809. Eur J Cancer. 2011;47:683-9.

12. Gnant MF, Mlineritsch $B$, Luschin-Ebengreuth $G$, et al. Zoledronic acid prevents cancer treatment-induced bone loss in premenopausal women receiving adjuvant endocrine therapy for hormone-responsive breast cancer: a report from the Austrian Breast and Colorectal Cancer Study Group. J Clin Oncol. 2007; 25:820-8.

13. Ellis GK, Bone HG, Chlebowski R, et al. Randomized trial of denosumab in patients receiving adjuvant aromatase inhibitors for nonmetastatic breast cancer. J Clin Oncol. 2008;26:4875-82.

14. Brufsky AM, Harker WG, Beck JT, et al. Final 5-year results of Z-FAST trial: adjuvant zoledronic acid maintains bone mass in postmenopausal breast cancer patients receiving letrozole. Cancer. 2012;118:1192-201

15. Hadji P, Aapro MS, Body JJ, et al. Management of aromatase inhibitor-associated bone loss (AIBL) in postmenopausal women with hormone sensitive breast cancer: joint position statement of the IOF, CABS, ECTS, IEG, ESCEO IMS, and SIOG.J Bone Onco. 2017;7:1-12.

16. GosS PE, Ingle JN, Pritchard KI, et al. Extending aromataseinhibitor adjuvant therapy to 10 years. N Eng/ J Med. 2016; 375:209-19

17. Lafage-Proust $\mathrm{MH}$, Roche $\mathrm{B}$, Langer $\mathrm{M}$, et al. Assessment of bone vascularization and its role in bone remodeling. Bonekey Rep. 2015;4:662.

18. Pierce SM, Recht A, Lingos TI, et al. Long-term radiation complications following conservative surgery (CS) and radiation therapy (RT) in patients with early stage breast cancer. Int J Radiat Oncol Biol Phys. 1992:23:915-23.

19. Cianferotti L, Bertoldo F, Carini M, et al. The prevention of fragility fractures in patients with non-metastatic prostate cancer: a position statement by the international osteoporosis foundation. Oncotarget. 2017;8:75646-63.

20. Dawson-Hughes B, Mithal A, Bonjour JP, et al. IOF position statement: vitamin $\mathrm{D}$ recommendations for older adults. Osteoporos Int 2010:21:1151-4.

21. Drake MT. Osteoporosis and cancer. Curr Osteoporos Rep.
2013;11:163-70

22. Coleman R, Cameron D, Dodwell D, et al. Adjuvant zoledronic acid in patients with early breast cancer: final efficacy analysis of the AZURE (BIG 01/04) randomised open-label phase 3 trial. Lancet Oncol. 2014:15:997-1006.

23. Coleman R, de Boer R, Eidtmann $\mathrm{H}$, et al. Zoledronic acid (zoledronate) for postmenopausal women with early breast cancer receiving adjuvant letrozole (ZO- FAST study): final 60-month results. Ann Oncol. 2013;24:398-405.

24. Gnant M, Pfeiler G, Dubsky PC, et al. Adjuvant denosumab in breast cancer (ABCSG-18): a multicentre, randomised, doubleblind, placebo-controlled trial. Lancet. 2015;386:433-43.

25. Rizzoli R, Body JJ, DeCensi A, et al. Guidance for the prevention of bone loss and fractures in postmenopausal women treated with aromatase inhibitors for breast cancer. an ESCEO position paper. Osteoporos Int. 2012;23:2567-76.

26. Early Breast Cancer Trialists' Collaborative Group (EBCTCG). Adjuvant bisphosphonate treatment in early breast cancer: meta-analyses of individual patient data from randomised trials. Lancet. 2015:386:1353-61.

27. Dhesy-Thind S, Fletcher GG, Blanchette PS, et al. Use of adjuvant bisphosphonates and other bone- modifying agents in breast cancer: a Cancer Care Ontario and American Society of Clinical Oncology Clinical Practice Guideline. J Clin Oncol. 2017;35:2062-81.

28. Gnant M, Pfeiler G, Dubsky PC, et al. The impact of adjuvant denosumab on disease-free survival: results from 3,425 postmenopausal patients of the ABCSG-18 trial. Cancer Res 2016;76(4 Suppl):S2-02.

29. Coleman RE, Finkelstein D, Barrios $\mathrm{CH}$, et al. Adjuvant denosumab in early breast cancer: First results from the international multicenter randomized phase III placebo controlled D-CARE study. J Clin Oncol. 2018:36(suppl 15):501 (abstr).

30. Black DM, Cummings SR, Karpf DB, et al. Randomised trial of effect of alendronate on risk of fracture in women with existing vertebral fractures. Lancet, 1996;348:1535-1541.

31. Cummings SR, Black DM, Thompson DE, et al. Effect of alendronate on risk of fracture in women with low bone density 
but without vertebral fractures: results from the Fracture Intervention Trial. JAMA. 1998;280:2077-82.

32. Black DM, Thompson DE, Bauer DC, et al. Fracture risk reduction with alendronate in women with osteoporosis: the Fracture Intervention Trial. J Clin Endocrinol Metab. 2000;85:4118-24.

33. Harris ST, Watts NB, Genant HK, et al. Effects of risedronate treatment on vertebral and nonvertebral fractures in women with is: a randomized controlled trial. JAMA.1999;282:1344-52

34. Reginster J, Minne HW, Sorensen OH, et al. Randomized trial of the effects of risedronate on vertebral fractures in women with established postmenopausal osteoporosis. Osteoporos Int. 2000;11:83-91.

35. McClung MR, Geusens P, Miller PD, et al. Effect of risedronate on the risk of hip fracture in elderly women. $N$ Engl J Med. 2001;344:333-40.

36. Chestnut CH, Skag A, Christiansen C, et al. Effects of oral ibandronate administered daily or intermittently on fracture risk in postmenopausal osteoporosis. J Bone Miner Res. 2004;19:1241-9.

37. Eisman JA, Civitelli R, Adami S, et al. Efficacy and tolerability of intravenous ibandronate injections in postmenopausal osteoporosis: 2-year results from the DIVA study. J Rheumatol. 2008;35:488-97.
38. Black DM, Delmas PD, Eastell R, et al. Once-yearly zoledronic acid for treatment of postmenopausal osteoporosis. N Eng/ J Med. 2007;356:1809-22.

39. Lyles KW, Colón-Emeric CS, Magaziner JS, et al. Zoledronic acid in reducing clinical fracture and mortality after hip fracture. $N$ Engl J Med. 2007:357:1799-809.

40. Cummings SR, San Martin J, McClung MR, et al. Denosumab for prevention of fractures in postmenopausal women with osteoporosis. N Engl J Med. 2009;361:756-65.

41. Bone $H G$, Wagman RB, Brandi ML,et al. 10 years of denosumab treatment in postmenopausal women with osteoporosis: results from the phase 3 randomised FREEDOM trial and open-label extension. Lancet Diabetes Endocrinol. 2017;5:513-23.

42. Jha S, Wang Z, Laucis $\mathrm{N}$, Bhattacharyya T. Trends in Media Reports, Oral Bisphosphonate Prescriptions, and Hip Fractures 1996-2012: An Ecological Analysis. J Bone Miner Res. 1996-2012: An Eco

43. Khosla S, Burr D, Cauley J, et al. American Society for Bone and Mineral Research. Bisphosphonate-associated osteonecrosis of the jaw: report of a task force of the American Society for Bone and Mineral Research. J Bone Miner Res. 2007;22:1479-91.

44. Shane E, Burr D, Abrahamsen B, et al. Atypical subtrochanteric and diaphyseal femoral fractures: second report of a task force of the American Society for Bone and Mineral Research. J Bone Miner Res. 2014;29:1-23.

45. Cummings SR, Ferrari S, Eastell R, et al. Vertebral fractures after discontinuation of denosumab: a post hoc analysis of the randomized placebo-controlled FREEDOM trial and its extension. J Bone Miner Res. 2018:33:190-8.

46. Freemantle N, Satram-Hoang S, Tang ET, et al.Final results of the DAPS (Denosumab adherence preference satisfaction) study: a 24-month, randomized, crossover comparison with alendronate in postmenopausal women. Osteoporos Int. 2012;23:317-26.

47. Leder BZ, Tsai JN, Jiang LA, Lee H. Importance of prompt antiresorptive therapy in postmenopausal women discontinuing teriparatide or denosumab: the denosumab and teriparatide follow-up study (DATA-Follow-Up). Bone. 2017:98:54-8.

48. McClung MR, Wagman RB, Miller PD, et al. Observations following discontinuation of long-term denosumab therapy. Osteoporos Int 2017;28:1723-32.

49. Lehmann T, Aeberli D. Possible protective effect of switching from denosumab to zoledronic acid on vertebral fractures. Osteoporos Int. 2017;28:3067.

50. (NCT02499237). Zoledronic Acid to Maintain Bone Mass After Denosumab Discontinuation (AfterDmab). 